\title{
PERFIL DOS PACIENTES ATENDIDOS EM UM HOSPITAL DE REFERENCIA AO TRAUMA EM CURITIBA: IMPLICAÇÕES PARA A ENFERMAGEM
}

\author{
Priscila Franco Di Credo ${ }^{1}$, Jorge Vinícius Cestari Felix ${ }^{2}$
}

\begin{abstract}
RESUMO: Objetivou-se conhecer a epidemiologia do trauma na realidade local, identificando o perfil dos pacientes, mecanismos de trauma, tempo de internação e desfecho. A pesquisa foi realizada em hospital de referência em trauma tendo como base os prontuários dos pacientes atendidos entre janeiro e junho de 2009. Os dados de interesse foram registrados em instrumento estruturado e elaborado para o estudo e analisados com auxílio de estatística descritiva. Verificou-se um perfil de homens jovens vítimas de acidentes de trânsito; em média os pacientes permaneceram internados no serviço de emergência por 54 horas, $57 \%$ foram internados e desses 7\% morreram. Mediante os dados sugere-se investimento em políticas públicas para a prevenção e redução de acidentes de trânsito e melhorias na organização dos serviços de saúde para que vítimas de trauma tenham garantido, quando indicado, hospitalização em unidade de internamento e não permaneçam longo período na unidade de emergência à espera de vaga. PALAVRAS-CHAVE: Serviços médicos de emergência; Trauma; Humanização da assistência; Enfermagem.

\section{PROFILE OF PATIENTS ATTENDED IN A CENTER OF EXCELLENCE IN TRAUMA HOSPITAL IN CURITIBA: IMPLICATIONS FOR NURSING}

\begin{abstract}
The aim was to understand the epidemiology of trauma in the local reality, identifying the profile of the patients, trauma mechanisms, time of hospitalization and outcome. The research was carried out in a hospital which is a center of excellence in trauma and based on the hospital notes of the patients attended during January - June 2009. The data of interest was recorded on an instrument structured and developed for the study, and analyzed with the aid of descriptive statistics. A profile of young men injured in traffic accidents was substantiated; on average, the patients were kept in the emergency service area for 54 hours, and $57 \%$ were admitted, of whom $7 \%$ died. The data suggests that it is appropriate to invest in public policies for the prevention and reduction of traffic accidents, and in improvements in the organization of health services such that trauma victims are guaranteed, when indicated, hospitalization on a ward rather than spending long periods in an emergency unit hoping for a place to become available. KEYWORDS: Emergency medical services; Trauma; Humanization of care; Nursing.

\section{PERFIL DE LOS PACIENTES ATENDIDOS EN UN HOSPITAL DE REFERENCIA AL TRAUMA EN CURITIBA: IMPLICACIONES PARA LA ENFERMERÍA}

RESUMEN: El objetivo del estudio fue conocer la epidemiología del trauma en la realidad local, identificando el perfil de los pacientes, mecanismos de trauma, tiempo de internación y desenlace. La investigación fue realizada en hospital de referencia en trauma utilizando como base los prontuarios de los pacientes atendidos entre enero y junio de 2009. Los datos de interés fueron registrados en instrumento estructurado y elaborado para el estudio y analizados con ayuda de estadística descriptiva. Se ha verificado un perfil de hombres jóvenes víctimas de accidentes de tránsito; en media los pacientes permaneceron internados en el servicio de emergencia por 54 horas, $57 \%$ fueron internados y de eses $7 \%$ murieron. Delante de los datos, se sugieren inversiones en políticas públicas para la prevención y reducción de accidentes de tránsito y mejorías en la organización de los servicios de salud para que víctimas de trauma tengan garantizada, cuando indicado, hospitalización en unidad de internamiento y no permanezcan largo periodo en la unidad de emergencia esperando plaza.

PALABRAS-CLAVE: Servicios médicos de emergencia; Trauma; Humanización de la asistencia; Enfermería.

\footnotetext{
${ }^{1}$ Enfermeira. Residente em Urgência e Emergência pelo Programa de Residência Multiprofissional do Hospital de Clínicas da Universidade Federal do Paraná-UFPR.

${ }^{2}$ Professor do Departamento de Enfermagem da UFPR. Doutorando em Ciências pelo Programa de Pós-Graduação em Fisiologia Humana do Instituto de Ciências Biomédicas da Universidade de São Paulo. Membro do Grupo de Pesquisa em Tecnologia e Inovação em Saúde-TIS.
} 


\section{INTRODUÇÃO}

O trauma é definido como o conjunto das perturbações - qualquer lesão de tecido, órgão ou parte do corpo - causadas subitamente por um agente físico de etiologia, natureza e extensão variadas e predominantemente de origens externas. A expressão sintomática e as manifestações clínicas são influenciadas por mecanismos de funcionamentos internos do próprio organismo. Desta forma, por ser proveniente da ação de agentes etiológicos conhecidos, por exigir atitudes e procedimentos terapêuticos específicos e acima de tudo por ser evitável, o trauma é considerado uma doença ${ }^{(1-2)}$. Representa assim, um sério problema social e comunitário com relevância crescente.

As transformações oriundas das mudanças sociais, políticas, econômicas e o acirrado crescimento urbano acarretaram problemas como a marginalização social, condições de desemprego e subemprego, levando ao aumento da violência nas grandes cidades. $\mathrm{O}$ desenvolvimento da indústria automobilística, associado à imprudência no trânsito e ao descumprimento da legislação em vigor, entre outros fatores elevaram o número de vítimas de trauma no trânsito ${ }^{(3-4)}$.

Ao analisar dados estatísticos relacionados às mortes por causas externas, é possível perceber que aumentam a cada ano, sendo que ao longo do tempo mostram ampla representatividade na atual configuração do padrão de mortalidade dos brasileiros ${ }^{(1-5)}$. Observa-se que o número de mortes tem índice expressivo na faixa etária entre 15 e 49 anos e o comportamento das mortes violentas demonstra elevada mortalidade masculina. Os homicídios e os acidentes de transporte representaram $62,5 \%$ do total de óbitos por causas externas no Brasil na última década. Nos homicídios, a mortalidade masculina chega a abranger atualmente 12 homens para cada mulher na faixa etária de 20 a $29 \operatorname{anos}^{(5)}$.

O trauma causa importantes consequências sociais e econômicas, pois além das lesões poderem ocasionar óbito ou incapacidade, temporária ou permanente. $\mathrm{O}$ alto custo com a recuperação e a piora da qualidade de vida são fatores que preocupam o Sistema Único de Saúde ${ }^{(6)}$. Essa epidemiologia tem impelido os serviços de atendimento de emergência no Brasil e em todo mundo a se organizarem para atender às cinco grandes vertentes do controle ao trauma: prevenção, atendimento pré-hospitalar, atendimento intra-hospitalar, reabilitação e plano de atendimento a catástrofes e grandes desastres ${ }^{(7)}$.

Atualmente, os serviços de atendimento pré-hos- pitalar na cidade de Curitiba são: o Serviço de Atendimento Móvel de Urgência (SAMU-192) da Prefeitura Municipal, e o Serviço Integrado de Atendimento ao Trauma (SIATE-193) do Corpo de Bombeiros. O SIATE, desde 1990 ao iniciar suas atividades, atendia tanto traumas quanto às emergências clínicas; em 2004 com a implantação do SAMU, ficou responsável apenas pelo atendimento a traumas. O SAMU desde então presta atendimento de urgência e emergência clínica e traumática em residências, locais de trabalho e vias públicas $^{(8)}$.

Neste contexto este estudo teve por objetivo identificar a epidemiologia do trauma na realidade local de um hospital referência da cidade de Curitiba.

\section{MÉTODO}

O estudo se caracterizou como transversal, descritivo de abordagem quantitativa. A pesquisa foi realizada de agosto a novembro de 2009 em um hospital público de referência em trauma na cidade de Curitiba-PR, tendo como base de dados os prontuários dos pacientes atendidos no Pronto-Socorro, no período de janeiro a junho de 2009. O estudo foi aprovado pelo Comitê de Ética em Pesquisa do hospital em questão sob o número 107/2009. Todos os aspectos éticos para realização de pesquisa em seres humanos foram observados, seguindo a Resolução 196/96 do Conselho Nacional de Saúde e complementares.

Delimitou-se, como critério de inclusão para a coleta de dados os prontuários dos pacientes atendidos no Pronto-Socorro pela Sala de Atendimento Avançado (SAV), ou seja, vítimas graves de trauma que necessitavam de assistência imediata. Dos 1.255 prontuários registrados no referido local de pesquisa, 801 foram analisados de forma randômica. Obtivemos, portanto, um grupo amostral de $63 \%$ do total, o que caracterizou de forma fidedigna a população em estudo ${ }^{(9)}$.

Através de um instrumento elaborado pelos autores, foram coletados dados referentes ao sexo, idade, ao mecanismo de trauma com as lesões apresentadas, os serviços de atendimento pré-hospitalar que atenderam e transportaram as vítimas, os dias de permanência no Pronto-Socorro, os dias de permanência no hospital do estudo e o desfecho desse atendimento. Considerouse "dias de internamento/permanência no serviço de emergência" o período em que o paciente ficou neste até o desfecho caracterizado por: conseguir leito em outra unidade do hospital, receber alta, vir a óbito ou ser transferido para outra instituição de saúde. 
No período de janeiro a junho de 2009, 31.240 pessoas foram atendidas pelo Serviço de Emergência do Hospital em estudo, totalizando a média de 5.206 atendimentos ao mês e 172 atendimentos ao dia. No total foram 3.276(10,5\%) internações, com média de 546 internações ao mês e 18 internações ao dia.

A maioria dos pacientes atendidos (89\%) recebeu alta e menos de $1 \%$ evoluíram para óbito ou foram removidos para outra instituição. Na SAV foram realizados $15 \%(4.656)$ dos atendimentos. Destes, $27 \%(1.255)$ dos pacientes permaneceram internados, o que correspondeu a $38,3 \%(1.255)$ dos 3.276 pacientes atendidos no serviço de emergência e que necessitaram de internação. Esta foi a população delimitada do estudo que atendeu aos critérios de inclusão. Dos 1.255 pacientes internados, a amostra foi de $801(63,8 \%)$ prontuários analisados de forma aleatória, sendo que destes, $1.5 \%(12)$ eram pacientes atendidos por de emergência clínica.

Estes dados foram analisados e apresentados com base na estatística descritiva; utilizou-se os programas Microsoft Excel e GraphPad Prism 5 para elaboração dos gráficos e tabelas.

\section{RESULTADOS}

Na identificação do perfil dessa população, observou-se a prevalência de 78\%(624) do sexo masculino. Houve maior incidência dos traumas 65,16\%(522), entre as faixas etárias de 15 a 44 anos, sendo esta composta de $85,63 \%(447)$ de homens e $14,37 \%(75)$ de mulheres. Apenas nas idades acima de 75 anos o trauma em mulheres foi mais representativo do que nos homens. Os principais mecanismos de trauma na amostra do estudo foram os acidentes de trânsito, as quedas e a violência, representando 50,3\%(403), 22,5\%(181), e $21,2 \%(170)$ respectivamente (Tabela 1$)$.

Entre os homens, os acidentes de trânsito foram o principal mecanismo de trauma, 52,7\%(331), seguido pela violência $25,6 \%(161)$ e quedas $15,4 \%(97)$. Já entre as mulheres a queda teve maior prevalência com $48,5 \%$ (84), seguida pelos acidentes de trânsito $41,6 \%$ (72) e violência
5,2\%(09). Considerou-se "violência" a junção dos casos de agressão física com os casos de ferimento por arma de fogo (FAF) e por arma branca (FAB). Como "Outros Traumas" 4,4\%(35) têm-se a junção de mecanismos de traumas que não ocorreram com uma frequência significativa para serem considerados separadamente, como queimaduras, afogamentos, empalamentos, soterramentos, queda de objeto sobre o indivíduo, acidentes de trabalho, entre outros (Gráfico 1).

A distribuição dos três principais mecanismos de trauma entre as faixas etárias revelou que tanto as vítimas de acidentes de trânsito quanto as vítimas de violência tiveram maior representatividade entre 15 e 29 anos,

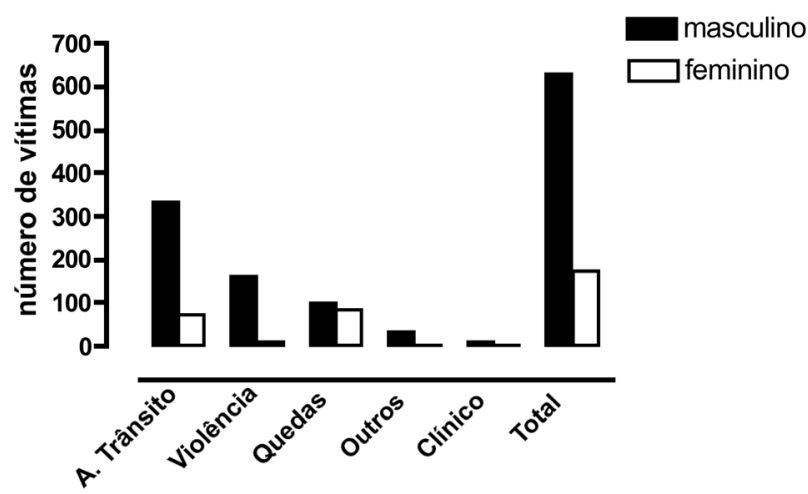

Gráfico 1- Mecanismo de tauma segundo o sexo da vítima. Curitiba, 2009

com 46,9\%(189) e 56,4\%(96) respectivamente, seguida pela faixa etária dos 30 aos 44 anos, com 26,5\%(107) e $34,6 \%(59)$ cada. As vítimas de quedas se encontraram em sua maioria entre os 30 a 59 anos com 40,8\%(74), sendo embriaguez, crises convulsivas e acidentes de trabalho os grandes responsáveis pelas mesmas (Tabela 1). A faixa etária acima dos 60 anos (terceira idade) ficou em segundo lugar com 34,6\%(63). Nos acidentes de trânsito, as colisões e os atropelamentos foram as principais causas de trauma, sendo que os motociclistas representaram aproximadamente $60 \%(240)$ das vítimas.

No tocante ao atendimento das vítimas e transporte ao serviço de emergência, $75 \%$ foram realizados pelo SIATE; $11 \%$ pelo SAMU; $5 \%$ por ambulâncias de prefeituras; $4,5 \%$ por carro particular; $3,5 \%$ por outros

Mec. de Trauma

Idade (anos)

\begin{tabular}{lllllllll}
\hline & $\mathbf{0 - 1 4}$ & $\mathbf{1 5 - 2 9}$ & $\mathbf{3 0 - 4 4}$ & $\mathbf{4 5 - 5 9}$ & $\mathbf{6 0 - 7 4}$ & $\mathbf{7 5 - 8 9}$ & $\mathbf{> 9 0}$ & Total \\
\hline Ac. Trânsito & $9,9 \%(40)$ & $46,9 \%(189)$ & $26,5 \%(107)$ & $10,6 \%(43)$ & $3,3 \%(13)$ & $2,8 \%(11)$ & 0 & 403 \\
Quedas & $13 \%(23)$ & $11,6 \%(21)$ & $16,5 \%(30)$ & $24,3 \%(44)$ & $13,7 \%(25)$ & $16,5 \%(30)$ & $4,4 \%(8)$ & 181 \\
Violência & $1,8 \%(3)$ & $56,4 \%(96)$ & $34,6 \%(59)$ & $5,4 \%(9)$ & $1,8 \%(3)$ & 0 & 0 & 170 \\
\hline
\end{tabular}

Figura 1 - Principais mecanismo de trauma segundo a faixa etária das vítimas. Curitiba, 2009 
serviços privados de atendimento pré-hospitalar; e $1 \%$ pela Polícia Militar. Dos 801 pacientes que chegaram ao serviço de emergência, 59(7,36\%) evoluíram para óbito após a internação. Destas vítimas, 84,7\%(50) eram do sexo masculino e 35,6\%(21) encontrava-se na faixa etária entre 15 e 29 anos. Em relação aos óbitos, mais da metade foram de vítimas de acidente de trânsito; a segunda causa de morte foi a violência, com 30,5\% (18) e, neste caso, a violência foi representada na totalidade por ferimento por arma de fogo. Quanto à faixa etária e o sexo, 67,8\%(40) dos homens morreram na idade produtiva, ou seja, nas faixas etárias de 15 a 59 anos. Já as mulheres apresentaram maior número de óbitos na faixa etária dos 75 aos 89 anos $44,5 \%(04)$ e as quedas foram responsáveis pela maioria dos óbitos nesses indivíduos 44,5\%(04).

Quanto à porcentagem de óbitos em cada faixa etária conforme o mecanismo de trauma, identificou-se que na faixa de 0 a 14 anos todas as mortes ocorreram em consequência dos acidentes de trânsito. Dos 15 aos 74 anos de idade, os acidentes de trânsito stambém foram a principal causa de morte, seguidos pelos ferimentos por armas de fogo (violência). Na faixa dos 75 aos 89 as quedas foram as responsáveis pela maioria dos óbitos, seguida pelos acidentes de trânsito, que foram representados principalmente pelos atropelamentos. Acima dos 90 anos, as quedas e os acidentes de trânsito apresentaram a mesma prevalência (Gráfico 2).

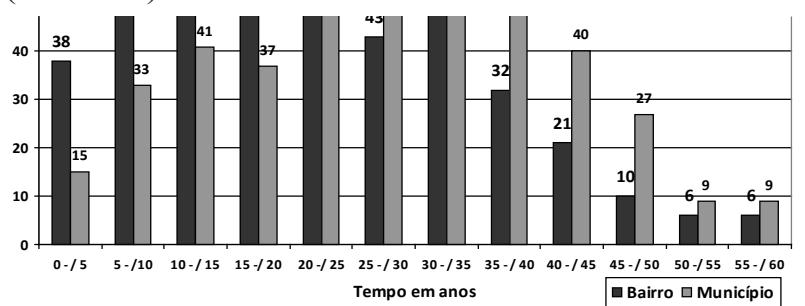

Gráfico 2- Relação entre os mecanismos de trauma e a faixa etária das vítimas fatais. Curitiba, 2009

As lesões mais frequentes foram as fraturas seguidas pelos traumatismos crânio-encefálicos. Porém, é importante destacar que embora existam lesões com menor incidência, como as lesões medulares, lacerações, esmagamentos e amputações, são estas de grande importância devido à gravidade das sequelas que provocam.

Com relação aos dias de internamento no serviço de emergência, observou-se que a média de permanência dos pacientes foi de dois dias e seis horas ou 54 horas. Os dados revelam que $67 \%(537)$ dos pacientes permaneceram mais de um dia (24 horas) internados no serviço de emergência. Quanto ao desfecho do atendimento, 56\%(449) dos pacientes foram encaminhados para outra unidade do hospital para continuar o tratamento; 39\%(312) receberam alta no pronto-socorro, e 4,5\%(36) vieram a óbito no período de internação no próprio serviço de emergência, a maioria em menos de 48 horas.

Quanto ao período total de internamento hospitalar, a média de permanência foi de 6,2 dias. Dos pacientes que foram encaminhados para outra unidade, $71 \%$ (321) foram para a clínica cirúrgica, sendo a grande maioria pacientes da ortopedia; $17 \%(74)$ para a pediatria e $12 \%(55)$ para a Unidade de Terapia Intensiva. Quanto ao desfecho hospitalar após o período total de internamento, $92 \%$ dos pacientes receberam alta, $7 \%$ foram à óbito e $1 \%$ foram transferidos para outra instituição de saúde.

\section{DISCUSSÃO}

Os resultados mostraram um perfil de pacientes jovens do sexo masculino, em idade produtiva, vítimas de acidentes de trânsito, a maioria condutores de motocicleta. Este perfil vem de encontro com os dados de 2008 do ministério da saúde quanto às causas externas por internação na população jovem (15 a 39 anos) ${ }^{(10)}$. Nota-se ainda, a expressividade da violência pelo grande número de ocorrências e mortes nesta mesma faixa etária.

$\mathrm{Na}$ amostra analisada, os principais mecanismos de trauma foram os acidentes de trânsito, as quedas e a violência. Já os dados nacionais de 2008 apresentam as quedas como principal mecanismos de trauma, seguidas pelos acidentes automobilísticos e agressões ${ }^{(10)}$.

O acometimento de homens jovens talvez seja influenciado pelo comportamento social e cultural. Pela tendência de serem mais agressivos no trânsito e pelas características próprias da idade tais como a impulsividade, imaturidade, a busca de sensações intensas, pouca experiência para dirigir, a motivação e influência do grupo de amigos e o comportamento delinquente ${ }^{(11-12)}$. Entre as mulheres, os principais mecanismos de trauma e de morte foram as quedas, seguidas pelos acidentes de trânsito. A faixa etária jovem também foi a mais atingida, porém, a da terceira idade teve maior número de óbitos.

Nesta população mais idosa, as quedas e os atropelamentos se destacaram. São inúmeros os fatores característicos dessa faixa etária que contribuem para a ocorrência desses mecanismos de trauma. Por exemplo, os problemas visuais e neurológicos, deficit de atenção, o declínio da função mental, fraqueza muscular e esquelética, características da idade e uso de substâncias psicoativas. Vários estudos demonstram relação entre o uso de medicações e as quedas, principalmente quanto ao uso de polifármacos ${ }^{(13)}$.

As colisões e os atropelamentos se destacaram entre 
os acidentes de trânsito, indo de encontro às estatísticas nacionais de 2008, sendo os condutores de motocicleta as principais vítimas no trânsito ${ }^{(9)}$. Pode-se relacionar o grande número de acidentes envolvendo motos com o número de motocicletas circulantes. Esse fenômeno acontece na maior parte das cidades do país, haja vista que estes veículos vêm ganhando cada vez mais aceitação e aprovação da população pela relação custo/benefício e também, pela grande demanda de serviços de entrega rápida via motoboy, que acabam exigindo menor tempo nas entregas e com isso ocasiona maior risco no trânsito ${ }^{(14)}$.

Quanto à ocupação do serviço de emergência, a pesquisa revelou que a média foi de 18 pacientes por dia, o que é três vezes maior do que a capacidade de leitos de observação deste serviço. Além disso, mais da metade dos pacientes permaneceu acima de 48 horas no local, com uma média de 54 horas. Observamos no serviço que este fato ocorreu devido à falta de leitos/ vaga nas unidades de internação do hospital. Acreditamos que isto resulta em sobrecarga de trabalho da equipe de enfermagem, visto que precisa atuar nas novas emergências que chegam e dar continuidade à assistência aos pacientes graves que aguardam vaga. Esta situação oferece riscos ao paciente e à própria equipe, considerando que o seu trabalho neste contexto já é demasiadamente intenso e estressante.

Mediante a dificuldade de transferência desse pacientes que necessitam de internação, ocorre ainda a superlotação do serviço de emergência pela realidade observada neste serviço, os pacientes permanecem em locais inadequados ao seu quadro de saúde, aguardando uma vaga de leito na instituição. Alguns pacientes graves, sedados e intubados que necessitam de assistência avançada, monitorização e vigia constantes, permanecem na SAV aguardando disponibilidade de vaga na UTI. Segundo o Ministério da Saúde, os serviços de pronto-socorro dispõem apenas de leitos de observação hospitalar, onde o paciente deve permanecer no hospital por um período menor que 24 horas, para fins de diagnósticos ou tratamento ${ }^{(15-16)}$.

Em relação ao desfecho do atendimento no serviço de emergência, a maioria das vítimas foi encaminhada para outras unidades do hospital, na maioria das vezes após um período superior a 48 horas, e a clínica cirúrgica foi a que recebeu o maior número desses pacientes, principalmente para cirurgias ortopédicas, devido a grande incidência de fraturas entre as lesões das vítimas.

O período de internamento hospitalar teve uma média de 6,2 dias, com o máximo de 94 dias. Quanto ao desfecho hospitalar, o número de óbitos foi pequeno, porém significativo no sexo masculino e nas faixa-etárias de 15 a 44 anos.
Além dos acidentes de trânsito, os ferimentos por arma de fogo também se mostraram expressivos, sendo a segunda causa de morte nesta mesma população. A violência crescente,

pelo número de vítimas e a magnitude de seqüelas orgânicas e emocionais que produz, adquiriu um caráter endêmico e se converteu num problema de saúde pública em vários países ${ }^{(17)}$.

Todo esse fenômeno conflitivo, causador de agravos à saúde e morte, se anuncia pela denominada transição epidemiológica para a qual contribuem as causas externas ${ }^{(4)}$. Mediante os dados obtidos nesta pesquisa e os resultados encontrados em outros estudos, acredita-se que os cenários da atenção às emergências e urgências nos serviços públicos no Brasil talvez não estejam preparados ou adaptados à atual transição epidemiológica e ainda enfrentam muitas dificuldades. Demanda-se maior investimento do Estado para o atendimento adequado das vítimas de trauma, políticas públicas efetivas que possam reduzir os índices alarmantes e, consequentemente, a morbi-mortalidade associada.

\section{CONCLUSÕES}

O perfil geral das vítimas de trauma da população analisada neste estudo não corresponde ao perfil geral nacional que é de população idosa, masculina, vítima de quedas. Porém, no que diz respeito ao perfil do trauma na população jovem apresentou concordância ao encontrado na literatura. Os acidentes de trânsito e a violência continuam a crescer, a afetar e a matar principalmente a população masculina jovem e economicamente ativa. Isto reflete alto custo para a sociedade e para o Sistema Único de Saúde.

Acreditamos que há necessidade de maior investimento na área (recursos humanos e infra-estrutura) e execução de políticas públicas efetivas voltadas para a prevenção e redução dos números de acidentes de trânsito, da violência $\mathrm{e}$, consequentemente, da morbi-mortalidade.

\section{REFERÊNCIAS}

1. Neto JB, Gomes EGA. Etilogia do Trauma. In: Freire E. Trauma: a doença do século. Rio de Janeiro: Atheneu; 2001. p. $403-20$

2. Mattos ACJ, Bousquat DA. Prevenção e ação no trauma, 2002. [acesso em 16 jun 2011] Disponível: www.saudebrasilnet.com.br/premios/saude/premio2/ trabalhos /056.pdf. 
3. Santos Júnior JCM. Instituto de Medicina: trauma - problema de saúde pública para o Século XXI. 2004. [acesso em 15 jun 2009] Disponível: www. grupodetrauma.com.br.

4. Minayo MCS. Caminhos do pensamento epistemologia e método. Rio de Janeiro: Fiocruz; 2002.

5. Sullivan J. Armas de fogo e saúde pública. 2002 In: DIAS, S.A. As representações da trajetória do atendimento de emergência para a vítima de trauma. [dissertação] Curitiba (PR): Universidade Federal do Paraná, 2004.

6. Gennari TD, Koizumi MS. Determinação do nível de gravidade do trauma. Rev. saude publica. 1995;29(5):33341.

7. Parolin KFM, Oliveira BFM, Teixeira Jr EV. Trauma: atendimento pré-hospitalar. São Paulo: Ateneu; 2004.

8. Almoyna MM, Nitschke CS. Regulação médica dos serviços de atendimento médico de urgência. SAMU, 1999. [acesso em 26 jun 2009] Disponível: http:// neu.saude.sc.gov.br/arquivos/manual_de_regulacao_ medica_de_urgencia.pdf.

9. Dyniewicz AM. Metodologia da pesquisa em saúde para iniciantes. São Paulo: Difusão; 2007.

10. DATASUS. [Internet]. Informações de saúde epidemiológicas e morbidade: causas externas por internação, 2008. [acesso em 2 dez 2011]. Disponível: http://tabnet.datasus.gov.br

11. Andrade SM, Soares DA, Braga GP, Moreira JH, Botelho FMN. Comportamentos de risco para acidentes de trânsito: um inquérito entre estudantes de medicina na região sul do Brasil. Rev. assoc. méd. bras. 2003;4(40):439-44.

12. Panichi RMD, Wagner A. Comportamento de risco no trânsito: revisando a literatura sobre as variáveis preditoras da condução perigosa na população juvenil. Interam. psicol. 2006;40(2):159-66.

13. Silva FS, Oliveira SK, Moreno FN, Martins EAP. Trauma no idoso: casos atendidos por um sistema de atendimento de urgência em Londrina. Com. ciências saúde. 2008,19(3):207-4.

14. Santos AMR, Moura MEB, Nunes BMVT, Leal CFS, Teles JBM. Perfil das vítimas de trauma por acidente de moto atendidas em um serviço público de emergência. Cad. Saude Publica. 2008;24(8):1927-38.
15. Ministério da Saúde (BR). Centro de Documentação do Ministério da Saúde Terminologia básica em saúde. Brasília, 1985.

16. Brasil. Portaria $n^{\circ} 737 /$ GM, de 16 de maio de 2001. Aprova a política nacional de redução da morbimortalidade por acidentes e violências. Diário Oficial da República Federativa do Brasil. Brasília, $2001, n^{\circ} 96$.

17. Organización Panamericana de la Salud Resolución XIX: Violencia y Salud. Washington; 1993. 